\title{
Regional patterns of myocardial sympathetic denervation in dilated cardiomyopathy: an analysis using carbon-11 hydroxyephedrine and positron emission tomography
}

\author{
F Hartmann, S Ziegler, S Nekolla, M Hadamitzky, M Seyfarth, G Richardt, M Schwaiger
}

\begin{abstract}
Objective-To assess presynaptic function of cardiac autonomic innervation in patients with advanced congestive heart failure using positron emission tomography (PET) and the recently developed radiolabelled catecholamine analogue carbon-11 hydroxyephedrine (HED) as a marker for neuronal catecholamine uptake function.
\end{abstract}

Design and patients-29 patients suffering from dilated cardiomyopathy with moderate to severe heart failure were compared with eight healthy controls. Perfusion scan was followed by HED dynamic PET imaging of cardiac sympathetic innervation. The scintigraphic results were compared with markers of disease severity and the degree of sympathetic dysfunction assessed by means of heart rate variability.

Results-In contrast to nearly normal perfusions, mean (SD) HED retention in dilated cardiomyopathy patients was abnormal in 64 (32)\% of the left ventricle. Absolute myocardial HED retention was $10.7(1.0) \% / m i n$ in controls $v 6.2(1.6) \%$ / min in dilated cardiomyopathy patients $(p<0.001)$. Moreover, significant regional reduction of HED retention was demonstrated in apical and inferoapical segments. HED retention was significantly correlated with New York Heart Association functional class $(r=-0.55$, $\mathbf{p}=0.002)$ and ejection fraction $(r=0.63$, p $<0.001$ ), but not, however, with plasma noradrenaline concentrations as well as parameters of heart rate variability.

Conclusions-In this study, using PET in combination with HED in patients with dilated cardiomyopathy, not only global reduction but also regional abnormalities of cardiac sympathetic tracer uptake were demonstrated. The degree of abnormality was positively correlated to markers of severity of heart failure. The pathogenetic mechanisms leading to the regional differences of neuronal damage as well as the prognostic significance of these findings remain to be defined.

(Heart 1999;81:262-270)

Keywords: heart failure; dilated cardiomyopathy; C-11 hydroxyephedrine; positron emission tomography; sympathetic nervous system
Neurohumoral activation is a common finding in patients with congestive heart failure. $.^{1-5} \mathrm{Sev}-$ eral alterations of the cardiac sympathoadrenergic system have been reported, ${ }^{6}$ all of them resulting in a reduction of cardiac responsiveness to $\beta$ adrenergic stimulation. ${ }^{7}$ Postsynaptic $\beta_{1}$ adrenoceptor density is reduced, ${ }^{89}$ whereas the concentrations of inhibiting $\mathrm{Gi} \alpha$ proteins are raised. ${ }^{10-12}$ These findings could partially be induced by the chronically raised plasma noradrenaline concentrations reported in these patients. ${ }^{1-5}$ In addition to that mechanism, cardiac noradrenaline turnover is increased. Sympathetic activation in chronic congestive heart failure may lead to increased noradrenaline release with increased spillover to plasma and subsequent tissue depletion. ${ }^{13-15}$ It is uncertain whether impaired neuronal reuptake acts as a relevant additive mechanism. Recently, in myocardial tissue from patients suffering from terminal congestive heart failure, Bohm et al found a reduction in noradrenaline uptake sites. But information on this issue is still limited and inconsistent. ${ }^{1617}$

Imaging of cardiac sympathetic innervation was first carried out in the 1980s with the introduction of I-123 metaiodobenzylguanidine (I-123 MIBG) and single photon emission

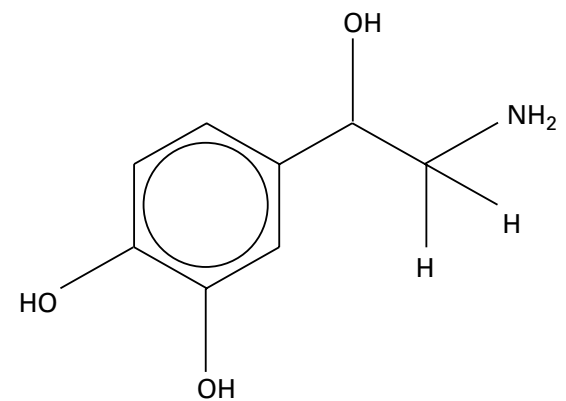

Noradrenaline

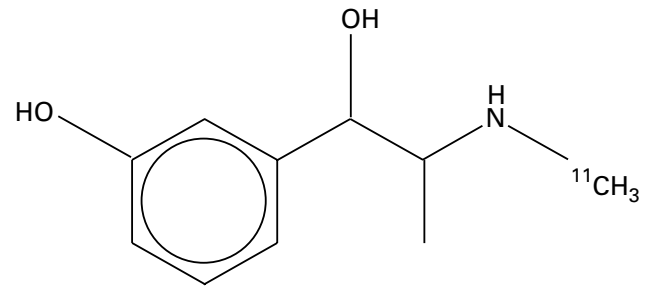

C-11 meta-hydroxyephedrine (HED)

Figure 1 Chemical structures of noradrenaline and C-11 hydroxyephedrine (HED). 


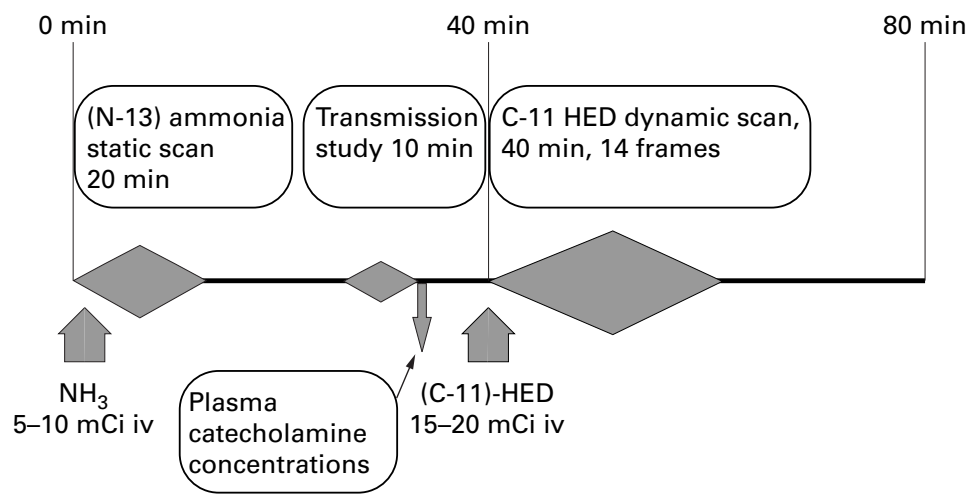

Figure 2 Imaging protocol. After positioning in the tomograph, a bolus injection of 185-370 MBq (5-10 mCi) of $\mathrm{N}-13$ ammonia was followed by a 20 minute static acquisition, commencing three minutes after injection. Then a 10 minute transmission study was acquired. After waiting one hour for $N-13$ decay, neuronal imaging of the heart was performed after bolus injection of $740 \mathrm{MBq}(20 \mathrm{mCi})$ of $\mathrm{C}-11$ hydroxyephedrine, with a subsequent 40 minute dynamic data acquisition in frame mode (14 frames) to determine tracer activity in both blood and myocardium.

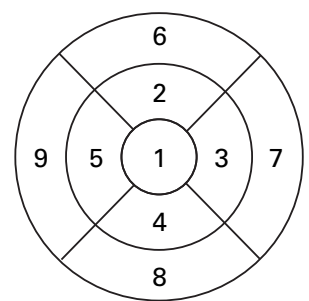

Figure 3 Scheme of the polar map areas and the corresponding left ventricular regions. 1 , apical; 2, anterior apical; 3, lateral apical; 4 , inferior apical; 5, septal apical; 6, anterior basal; 7 , lateral basal; 8, inferior basal; 9 , septal basal. computed tomography (SPECT) techniques. Recently, positron emission tomography (PET) imaging, using the newly developed synthetic catecholamine analogue carbon-11 (C-11) hydroxyephedrine (HED) (fig 1) provides quantitative means for non-invasive investigation of presynaptic neuronal function. ${ }^{18}$ After intravenous injection HED clears rapidly from the blood pool and is avidly retained in myocardium, with heart to blood ratio exceeding 5:1 within 10 minutes. Myocardial activity remains constant at about $80 \%$ of the peak activity for at least 60 minutes. ${ }^{18}$ The tracer is useful in the assessment of global and regional changes of cardiac sympathetic innervation under various clinical conditions, as shown in patients after cardiac transplantation, ${ }^{19}$ with diabetic autonomic neuropathy, ${ }^{20}$ or after myocardial infarction. ${ }^{21}$

This study aimed to assess cardiac autonomic innervation in patients suffering from advanced congestive heart failure, using HED as a marker for presynaptic neuronal uptake-1 activity, and to compare the scintigraphic findings with the severity of cardiac disease and with markers of sympathetic dysfunction, quantified by means of heart rate variability.

\section{Methods}

STUDY PATIENTS

Two groups of patients were investigatedeight healthy volunteers without any medical treatment served as control subjects and were compared with 29 patients suffering from idiopathic dilated cardiomyopathy, New York Heart Association (NYHA) class II-III. Idiopathic dilated cardiomyopathy was assumed in the absence of coronary heart disease, excluded

Table 1 Clinical characteristics of the study groups

\begin{tabular}{lclll}
\hline & \multicolumn{5}{c}{$\begin{array}{l}\text { Mean } \\
\text { (SD) age } \\
\text { (years) }\end{array}$} & $\begin{array}{l}\text { Sex } \\
(M / F)\end{array}$ & $\begin{array}{l}\text { Mean (SD) } \\
\text { LVEF (\%) }\end{array}$ \\
Group & Number & & & \\
\hline Healthy controls & 8 & $38(9)$ & $6 / 2$ & - \\
DCM patients & 29 & $46(11)$ & $26 / 3$ & - \\
NYHA II & 13 & $44(12)$ & - & $25(7)$ \\
NYHA III & 16 & $48(11)$ & - & $18(6)$ \\
\hline
\end{tabular}

LVEF, left ventricular ejection fraction; DCM, dilated cardiomyopathy; NYHA, New York Heart Association functional class. by coronary angiography, valvular heart disease, or any kind of secondary cardiomyopathy. Patients in stable clinical conditions, with a history of cardiomyopathy of more than six months' duration, were eligible for the study. Medical treatment of all patients consisted of diuretics, angiotensin converting enzyme inhibitor, and, if not contraindicated, digoxin. Medications known or suspected to interfere with neuronal uptake of catecholamine analogues, such as tricyclic antidepressants, were discontinued for more than two weeks before the patients were entered into the study. None of the patients was on $\beta$ blocker treatment before the scintigraphic examination. Patients with a history of diabetes mellitus ${ }^{20}$ or amiodarone treatment, which are suspected to interfere with HED kinetics, were excluded.

The study protocol was approved by the institutional ethics committee of the Technische Universität München. Written informed consent was obtained from all patients and control subjects before entering the study.

\section{CLINICAL EVALUATION}

Clinical examination was performed before the PET study and history of disease was recorded in all patients. Symptoms were described using NYHA functional class criteria. To determine resting ejection fraction, gated blood pool imaging was carried out in all patients. Plasma catecholamine concentrations were determined during PET. After a resting period of at least 30 minutes blood samples were obtained in supine position, from a needle placed in a large forearm vein at the beginning of the investigation. Blood sampling was performed without stoppage, using heparinised $5 \mathrm{ml}$ syringes, which were cooled on ice immediately. Plasma was separated using a cooled centrifuge for 10 minutes and stored at $-60^{\circ} \mathrm{C}$ until assay. Noradrenaline concentrations were determined using a high performance liquid chromatography method. ${ }^{22} 23$

To determine parameters of heart rate variability, ambulatory ECG tapes of patients with preserved sinus rhythm were replayed using a Zymed 1610 high speed Holter scanner with an automated analysis system trained to differentiate between normal and abnormal QRS complexes. All abnormal QRS complexes and their adjacent intervals, as well as phases of intermittent atrial fibrillation, were excluded from the calculation of heart rate variability. Cycle lengths differing less than $20 \%$ from the preceding sinus cycle length $(\mathrm{NN})$ were stored as R-R interval file for further evaluation. Time domain parameters of heart rate variability were calculated as: (1) standard deviation of all R-R intervals over 24 hours (SDNN); (2) standard deviation of the mean R-R interval for all five minute segments of a 24 hour recording (SDANN); (3) heart rate variability triangular index (HRVTrilnd), calculated by dividing the total number of $\mathrm{R}-\mathrm{R}$ intervals by the modal $\mathrm{R}-\mathrm{R}$ interval frequency; (4) square root of the mean of the sum of the squares of differences between adjacent $\mathrm{NN}$ intervals (RMSSD). ${ }^{24}$ 


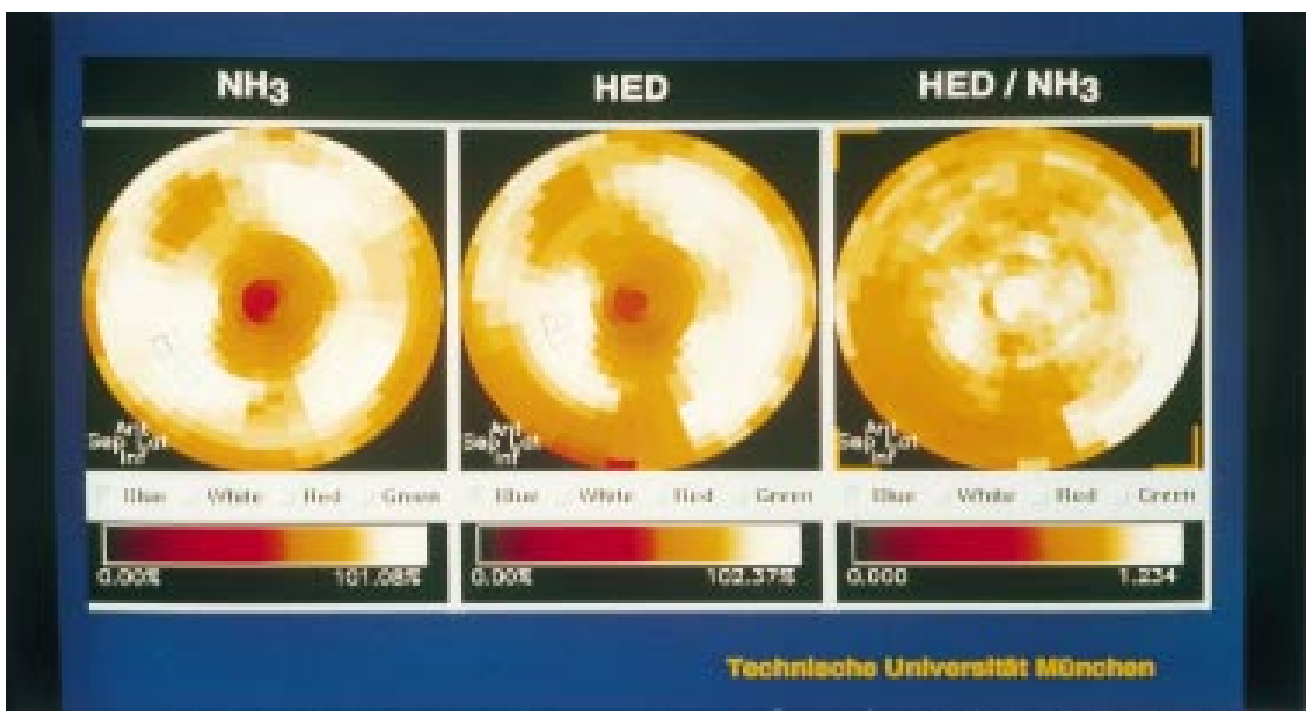

Figure 4 PET study of a 50 year old patient with moderate heart failure and a left ventricular ejection fraction of $30 \%$. Blood flow images showed a normal perfusion pattern, but HED retention index was reduced by $20 \%$ compared to normal values. The regional tracer distribution appeared normal on visual inspection with only a small region of abnormal tracer retention in the distal inferior left ventricle.

POSITRON EMISSION TOMOGRAPHY

The scintigraphic methods are described in detail elsewhere. ${ }^{18} 2021$ 25-29 Therefore, they are summarised briefly.

All PET studies were performed on a Siemens/CTI 95115 slice whole body tomograph. Imaging was carried out within four weeks of the initial clinical evaluation. Perfusion scan using nitrogen N-13 ammonia was combined with HED dynamic PET according to the following scheme (fig 2). Imaging was initiated with $185-370 \mathrm{MBq}(5-10 \mathrm{mCi})$ of $\mathrm{N}-13$ ammonia, injected intravenously, in order to evaluate myocardial perfusion at rest. A 10 minute static acquisition was performed, commencing three minutes after injection. Then a transmission study for subsequent attenuation correction of the emission data for attenuation was acquired using a retractable germanium-68 ring source. After waiting one hour for N-13 decay, neuronal imaging of the heart was performed after bolus injection of
$740 \mathrm{MBq}(20 \mathrm{mCi})$ of HED. Data were acquired dynamically in frame mode (14 frames) to determine tracer activity in both blood and myocardium.

IMAGE RECONSTRUCTION

HED and N-13 ammonia emission data were attenuation corrected and reconstructed by filtered back projection using a Hanning filter with cut off of 0.3 cycles/pixel. Images were reoriented to the long and short axes of the left ventricle using a SUN computer workstation. A semiquantitative approach was used to determine HED uptake both globally and regionally. A microcomputer based circumferential count profile analysis was performed on the original images of perfusion and HED retention with radial search for maximal activity in 36 sectors around the left ventricular wall. The results were depicted as polar maps of maximal tracer activity. HED retention index was determined by normalising myocar-

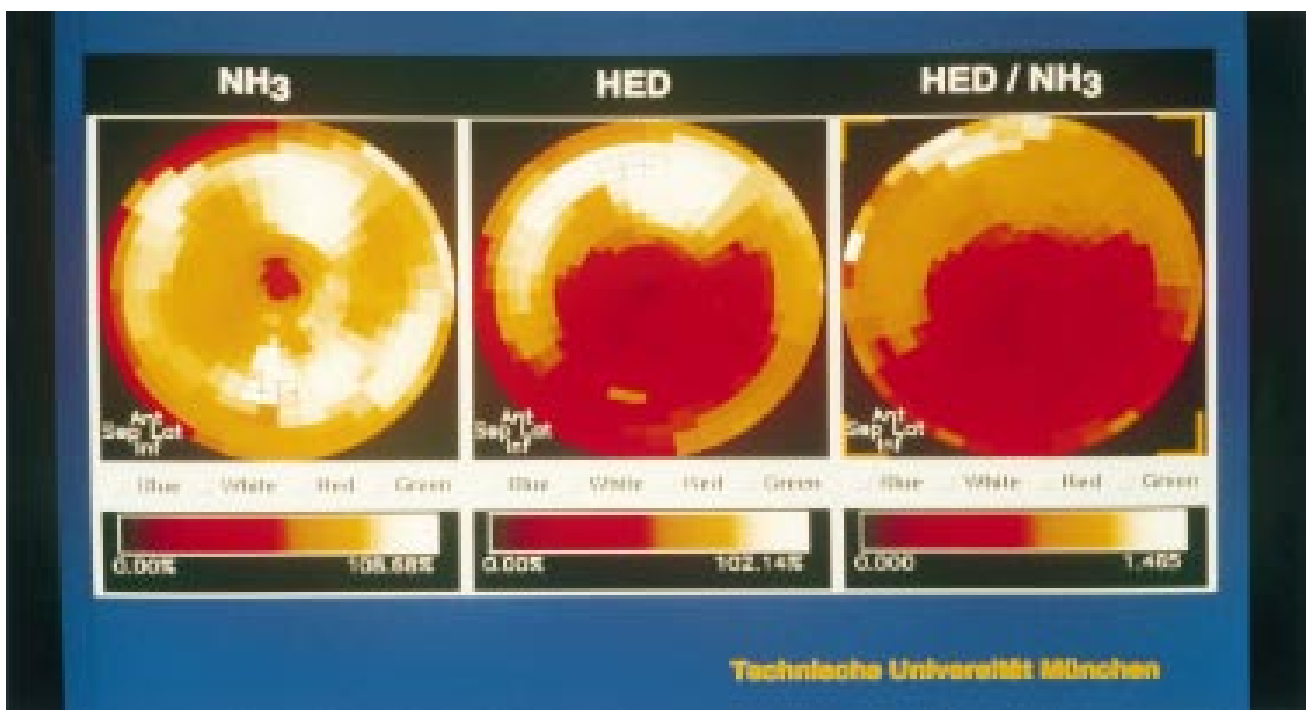

Figure 5 PET study of a 43 year old patient with severe heart failure and an ejection fraction of $16 \%$. Blood flow images again were normal, but HED images showed a regional reduction of tracer retention in the distal lateral and inferior wall. 


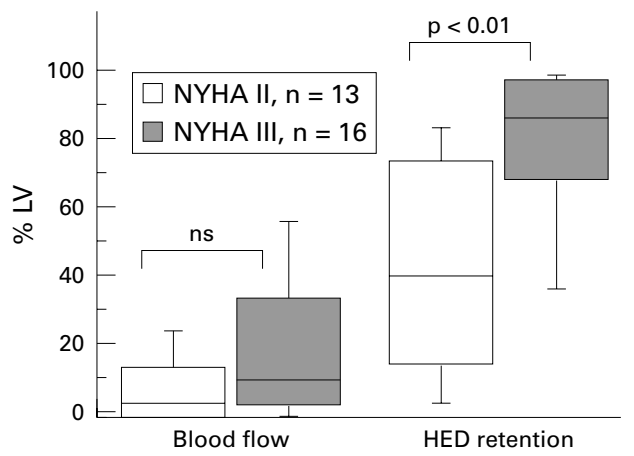

Figure 6 Extent of neuronal and blood flow inhomogeneities in dilated cardiomyopathy, expressed as percentage of polar map area $<2.5$ SD below control values ( $\%$ left ventricular). HED retention was significantly different in NYHA II v NYHA III patients $(p<0.01)$.

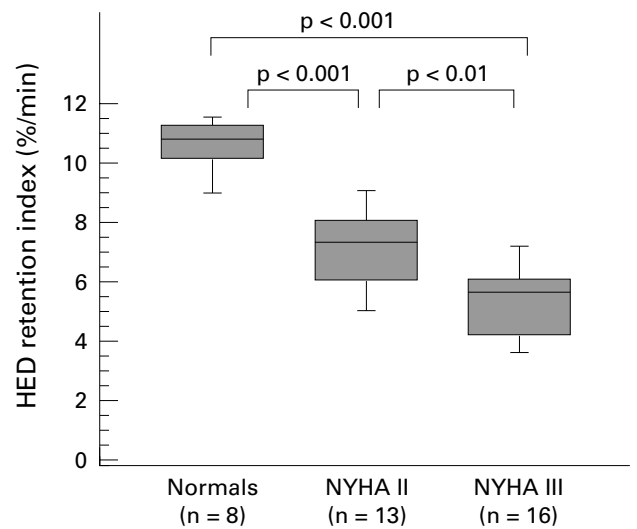

Figure 7 Absolute tissue retention index of C-11 HED at 40 minutes, normalised to the integral arterial input function, was significantly reduced in dilated cardiomyopathy patients. The degree of reduction corresponded to NYHA functional class.

dial tracer activity from $30-40$ minutes to the integral of the C-11 activity input function, which was determined from the area under the time-activity curve for a small region of interest $(3 \times 3-4 \times 4$ pixels $)$ placed in the centre of the left ventricular blood pool. The following formula was used: retention index (\%/

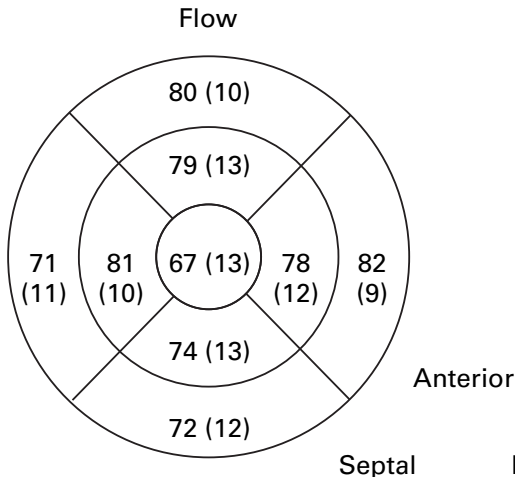

Septal

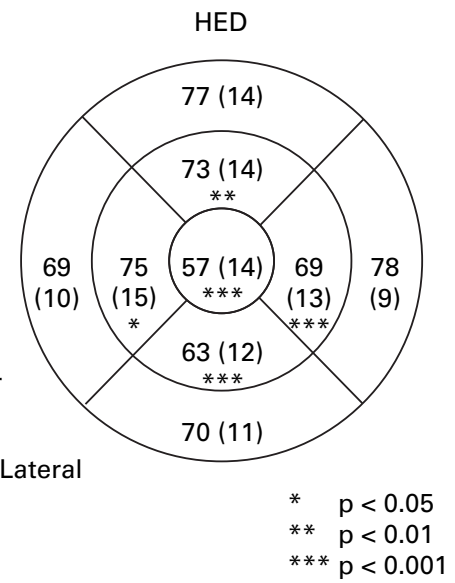

Figure 8 Maps of flow (left side) and C-11 HED retention (right side) in dilated cardiomyopathy patients. Regional tracer activity is expressed as a percentage of the individual maximum of each polar map. Despite a homogenous perfusion pattern, HED retention is reduced significantly (compared to the corresponding perfusion values) in the apical segments. $\min )=$ tissue counts between $30-40$ min $\div$ sum blood counts from time 0 to 40 $\min$.

The HED retention index of every segment was computed and displayed as polar coordinate map of regional tracer activity.

\section{HOMOGENEITY OF MYOCARDIAL TRACER}

RETENTION

The studies performed in healthy volunteers were averaged to form a database of normal values of HED retention. Individual patient polar maps were compared with the database. Values > 2.5 SD below database values were defined as abnormal. The extent of heterogeneity was expressed as the percentage of left ventricular map area $>2.5 \mathrm{SD}$ below database values.

REGIONAL PATTERN OF TRACER RETENTION

The polar maps were divided into nine regions, as depicted schematically in fig 3 . Within each region the tracer activity was expressed both absolutely and as a percentage of the maximal activity in the map.

Myocardial HED retention has been previously shown to be flow related. ${ }^{30}$ To avoid these limitations, polar maps of $\mathrm{N}-13$ ammonia and HED retention were normalised to the highest values $(3 \times 3$ pixels $)$ found in every data set. Then the relation between myocardial blood flow and HED retention was computed to determine the ratio of regional $\mathrm{HED} /$ flow counts for combined proximal, distal, and apical segments. Values below 1 represent regions with preserved perfusion but reduced HED retention.

\section{STATISTICAL METHODS}

Data are expressed as mean (SD). Differences in group means were compared using the Mann-Whitney non-parametric test for unpaired samples. The difference between flow and HED retention in the same patient was compared using the Student test for paired samples. The relation between clinical datafor example, resting ejection fraction, plasma noradrenaline concentrations, heart rate variability parameters, and HED retention-was compared by linear regression, computing the Pearson correlation coefficient $r$ and $t$ test for significance of the slope of the regression line. Significance was defined as $\mathrm{p}<0.05$.

\section{Results}

The baseline characteristics of the two groups are summarised in table 1 . Mean age was 38 (9) years in the control group, compared to 46 (11) years in the patients with dilated cardiomyopathy. Thirteen dilated cardiomyopathy patients were in NYHA functional class II (mean ejection fraction 25 (7)\%), 16 in NYHA functional class III (mean ejection fraction 18 (6)\%). Examples of PET images and the obtained polar maps of the circumferential count profile analysis are depicted for two patients (fig 4 and fig 5). 

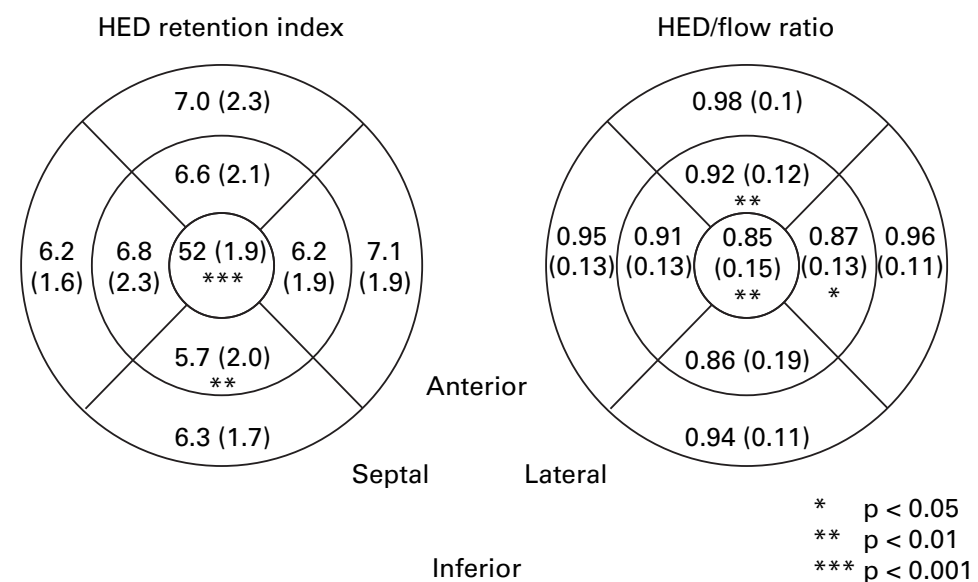

Figure 9 Regional C-11 HED retention index (left side) was progressively reduced from proximal to distal $(p<0.05$ in the inferoapical and $p<0.001$ in the apical segment). Regional HED/flow ratio (right side) in dilated cardiomyopathy patients. Values close to unity mean correspondence between flow and HED retention and were found in the basal segments. However, they were progressively reduced from basal to apical myocardium $(p<0.05$ in the apical and inferoapical segment).

INHOMOGENEITY OF HED RETENTION IN DILATED CARDIOMYOPATHY

$\mathrm{N}-13$ ammonia retention throughout the left ventricle was homogenous in all patients with dilated cardiomyopathy. Only 15 (20)\% of the left ventricular polar map area was $<2.5 \mathrm{SD}$ of the control values. In contrast HED retention images showed abnormalities of tracer retention in 64 (33)\% of polar map areas. The extent

Table 2 Correlation matrix for absolute values as well as inhomogeneity of $C-11 \mathrm{HED}$ retention and NYHA function class, left ventricular ejection fraction (LVEF), and plasma noradrenaline concentrations in dilated cardiomyopathy patients

\begin{tabular}{clll}
\hline & NYHA & LVEF & Noradrenaline \\
\hline $\begin{array}{c}\text { C-11 HED retention } \\
\text { (absolute) }\end{array}$ & $\begin{array}{l}0.551^{\star \star} \\
(\mathrm{n}=28)\end{array}$ & $\begin{array}{l}0.626^{\star \star \star} \\
(\mathrm{n}=28)\end{array}$ & $\begin{array}{l}0.251(\mathrm{~ns}) \\
(\mathrm{n}=20)\end{array}$ \\
$\begin{array}{l}\text { Inhomogeneity } \\
0.542^{\star \star}\end{array}$ & $\begin{array}{l}0.492^{\star \star} \\
(\mathrm{n}=28)\end{array}$ & $\begin{array}{l}0.150(\mathrm{~ns}) \\
(\mathrm{n}=28)\end{array}$ & $(\mathrm{n}=20)$
\end{tabular}

${ }^{\star \star} \mathrm{p}<0.01 ;{ }^{\star \star \star} \mathrm{p}<0.001$.

Table 3 Correlation matrix for HED retention and resting noradrenaline concentrations in dilated cardiomyopathy patients $(n=20)$

\begin{tabular}{lcccc}
\hline & HED-abs & HED $<2.5$ SD & Noradrenaline \\
\hline HED-abs & 0.907 & 0.320 \\
HED $<2.5 S D$ & $<0.001$ & 0.221 \\
Noradrenaline & 0.251 & 0.437 & \\
\hline
\end{tabular}

HED-abs, absolute C-11 hydroxyephedrine retention; HED < $2.5 \mathrm{SD}$, inhomogeneity of $\mathrm{C}-11$ hydroxyephedrine retention.

Table 4 Correlation matrix for HED retention and time domain parameters of heart rate variability in dilated cardiomyopathy patients

\begin{tabular}{cllll}
\hline & $S D N N$ & $S D A N N$ & RMSSD & HRVTrilnd \\
\hline $\begin{array}{c}\text { C-11 HED } \\
\text { retention }\end{array}$ & $\begin{array}{l}0.299 \\
(\mathrm{n}=16)\end{array}$ & $\begin{array}{l}0.225 \\
(\mathrm{n}=16)\end{array}$ & $\begin{array}{l}0.144 \\
(\mathrm{n}=16)\end{array}$ & $\begin{array}{l}0.437 \\
(\mathrm{n}=16)\end{array}$ \\
$\begin{array}{l}\text { Inholute }) \\
\begin{array}{l}0.393 \\
(\mathrm{n}=16)\end{array}\end{array}$ & $\begin{array}{l}0.228 \\
(\mathrm{n}=16)\end{array}$ & $\begin{array}{l}0.293 \\
(\mathrm{n}=16)\end{array}$ & $\begin{array}{l}0.508^{\star} \\
(\mathrm{n}=16)\end{array}$ \\
\hline
\end{tabular}

${ }^{\star} \mathrm{p}<0.05$.

SDNN, standard deviation of all R-R intervals over 24 hours; SDANN, standard deviation of the mean R-R interval for all five minute segments of a 24 hour recording; HRVTrilnd, heart rate variability triangular index; RMSSD, square root of the mean of the sum of the squares of differences between adjacent NN intervals. of regional retention abnormalities was significantly different $(\mathrm{p}<0.01)$ in NYHA II $v$ NYHA III patients (mean 44 (23)\% $v 78$ (24) $\%$ of polar map area) (fig 6). Perfusion was not different in both groups.

ABSOLUTE TISSUE RETENTION INDEX FOR HED Not only was inhomogeneity of HED retention enhanced in dilated cardiomyopathy patients. Absolute tissue retention of HED was reduced significantly compared to controls. Mean values for absolute tissue retention index for HED are shown in fig 7 for the control group (10.7 $(1.0) \% / \mathrm{min})$ and for patients with moderate $(7.2(1.7) \% / \mathrm{min})$ to severe $(5.5(1.5) \% /$ min) heart failure. The difference reached significance for both groups, compared to controls and to each other.

REGIONAL HED RETENTION IN DILATED CARDIOMYOPATHY

Maps of mean relative tracer retention, expressed as a percentage of the maximal activity in the map, are shown in fig 8 for both flow and HED studies. Flow studies showed homogenous tracer retention throughout all regions of the left ventricular map. HED retention in dilated cardiomyopathy patients, however, showed a regional pattern. Decreased HED retention was found especially in the distal segments and reached a maximum in the apical and distal inferior segment $(10 \%$ and $11 \%$ less than the corresponding flow values, $\mathrm{p}<0.001)$.

\section{HED/FLOW RATIOS}

In fig 9 absolute regional HED retention indices (left side) and the relation between myocardial blood flow and HED retention (right side) are shown for proximal, distal, and apical segments in dilated cardiomyopathy patients. Absolute regional HED retention index was progressively reduced from proximal to distal. The difference to each patient's mean values reached significance in the inferoapical $(-0.5 \%, \quad \mathrm{p}<0.01)$ and apical $(-1.0 \%$, $\mathrm{p}<0.001)$ segments. To avoid the limitation of flow dependence of HED retention, regional HED/flow ratios were computed. Values close to unity meant correspondence of perfusion and HED retention, and were obtained in all basal segments. However, there was a progressive reduction in HED/flow ratio in the apical segments. The lowest values were obtained in the apical $(0.85(0.15))$ and inferoapical $(0.86$ (0.19)) segments. The difference from the mean values of each patient reached significance in the apical $(-0.06, \mathrm{p}<0.001)$ and lateroapical $(-0.03, \mathrm{p}<0.05)$ segments.

RELATION BETWEEN ABSOLUTE REDUCTION AND INHOMOGENEITY OF HED RETENTION

Highly significant negative correlation was found between absolute HED retention and the inhomogeneity of tracer retention, expressed as a percentage of polar map area $<2.5$ SD of normals $(r=0.921, \mathrm{p}<0.001)$, indicating that both parameters reflect alterations of cardiac sympathetic innervation in dilated cardiomyopathy patients in a similar manner. 
RELATION BETWEEN CLINICAL PARAMETERS OF SEVERITY OF HEART FAILURE AND THE SCINTIGRAPHIC FINDINGS

In dilated cardiomyopathy patients absolute reduction as well as the inhomogeneity of $\mathrm{HED}$ retention was positively correlated to NYHA functional class $(r=0.55, \mathrm{p}=0.002$, and $r=0.54, \mathrm{p}=0.002$, respectively) and ejection fraction $(r=0.63, \mathrm{p}<0.001$, and $r=0.49$, $\mathrm{p}=0.007$ ) (table 2). No correlation was found with the plasma noradrenaline concentrations at rest, obtained in 20 cases during the PET investigation (table 3 ).

In 16 cases with preserved sinus rhythm Holter ECGs were available to determine heart rate variability. Weak correlation was detected between inhomogeneity of tracer retention and HRVTrilnd $(r=0.51, \mathrm{p}=0.04)$. No correlation was detected between HED retention and other time domain parameters of heart rate variability in this subset of patients (table 4 ).

\section{Discussion}

The scintigraphic approach of this study provides new insights into the changes of the cardiac sympathoadrenergic system in patients with progressive congestive heart failure. PET and HED retention in cardiac neuronal tissue was used as a marker for the integrity of sympathetic nerve terminals. Patients with advanced dilated cardiomyopathy demonstrate not only an absolute reduction of myocardial HED retention of about $42 \%$, but also a regional pattern of retention abnormalities. Lowest values of tracer activity were demonstrated in the inferior and apical segments. The reduction was independent of differences in tracer delivery as demonstrated by direct comparison to myocardial perfusion assessed by N-13 ammonia. However, a weak correlation was observed between myocardial HED activity and markers for the severity of heart failure-for example, resting ejection fraction and NYHA functional class-but not with resting noradrenaline concentrations and heart rate variability criteria.

DIFFERENT TRACERS FOR CARDIAC SYMPATHETIC NEURONAL FUNCTION

Previous studies using SPECT and I-123 MIBG, another noradrenaline analogue with high affinity to sympathetic neuronal tissue, for the evaluation of presynaptic sympathetic function showed pathological findings under different conditions of cardiac diseases. Pathological findings of MIBG imaging, reflecting functional alteration of sympathetic innervation, were first reported in heart failure dogs by Rabinovitch and colleagues, ${ }^{31}$ and in patients with dilated cardiomyopathy by Schofer, ${ }^{32}$ and Glowniak. ${ }^{33}$ I-123 MIBG myocardial retention was significantly reduced and heterogeneity in tracer distribution significantly increased. Relations between tracer retention, myocardial noradrenaline concentrations derived from endomyocardial biopsy samples, and left ventricular ejection fractions were demonstrated.

Methodical problems, however, limit the value of myocardial I-123 MIBG scintigraphy as a non-invasive method for the quantitative assessment of myocardial adrenergic nervous system in patients with idiopathic dilated cardiomyopathy. The application of SPECT technique results in restricted spatial resolution which makes the determination of regional differences difficult and quantification of tracer uptake impossible. Furthermore, interpretation of I-123 MIBG cardiac behaviour is complicated by a considerable amount of nonspecific tissue uptake, reflecting non-neuronal tracer deposition sites.

These limitations can be avoided using PET and HED, which, similar to I-123 MIBG, competes with noradrenaline for the energy dependent neuronal catecholamine transport system (uptake-1) and vesicular storage mechanisms. HED cardiac behaviour is characterised by a very rapid neuronal uptake via uptake-1, which is "flow limited". "Unlike noradrenaline, HED is not metabolised within the neuron by the monoamine oxidase. It is transported into vesicles by the monoamine transporter. In contrast to natural catecholamines, HED, because of its more lipophilic and less polar properties, rapidly leaks out of vesicles and diffuses across neuronal membranes back into interstitium. Thus, HED is dynamically recycled into the neuron, its kinetics being sensitive to the degree of sympathetic activation leading to increased competition with noradrenaline for transport at the neuronal transporter. ${ }^{34}$ Unlike I-123 MIBG, HED extraneuronal uptake is very low, as determined in desipramine treated rat hearts. ${ }^{34}$ Similar to I-123 MIBG, however, HED myocardial clearance rate does show a dependence on noradrenaline concentrations. ${ }^{25}{ }^{34}$

HED cardiac imaging in humans has been shown to be specific and sensitive for alterations of neuronal function. Clinical studies in patients after heart transplantation demonstrated complete abolition of tracer retention in the denervated donor heart in the early postoperative phase, whereas in the later period an increasing degree of HED retention was interpreted as reinnervation of the transplanted organ. ${ }^{19}$ Studies in patients after myocardial infarction undergoing thrombolytic treatment, ${ }^{27}$ and in patients with diabetic sympathetic neuropathy, ${ }^{20}$ showed an involvement of the cardiac sympathoadrenergic system in the disease process with localised defects of sympathetic innervation. However, to our knowledge, this is the first study using PET and HED in patients with congestive heart failure.

\section{MECHANISMS OF SYMPATHOADRENERGIC} DYSFUNCTION IN DILATED CARDIOMYOPATHY The regional pattern of neuronal dysfunction in dilated cardiomyopathy appears to be similar to the findings in patients suffering from progressive dysfunction of the autonomic innervation of the heart, associated with diabetic neuropathy. ${ }^{20}$ In both cases the possible mechanisms and underlying structural abnormalities leading to the process of neuronal damage are unknown. Possible aetiologies include functional impairment caused by metabolic abnormalities, neuronal ischaemia, and immune mediated injury. 
There are several possible explanations for a decrease in myocardial HED retention in dilated cardiomyopathy. Reduced HED delivery as a consequence of reduced myocardial blood flow could be ruled out by the normal perfusion scans. Competitive inhibition of reuptake, caused by raised circulating noradrenaline concentrations, may be another. However, no correlation was found between plasma noradrenaline concentrations and HED retention in this study, making this explanation unlikely. Increased sympathetic nerve firing rates in heart failure may be a further mechanism for competition between endogenous neuronal noradrenaline reuptake and HED uptake. The increased wall stress of a dilated left ventricle may result in metabolic abnormalities, leading to decreased activity of either or both the highly energy dependent uptake- 1 function and vesicular storage mechanism. The subsequent reduction of neuronal reuptake with lacking inactivation of noradrenaline in the synaptic cleft may play a key role in all subsequent changes of the sympathetic signal transduction pathway in heart failure, especially the postsynaptic desensitisation. Differentiation between uptake or storage abnormalities in the sympathetic nerve terminal is not possible using PET and HED. However, recently in vitro binding assays on myocardial tissue of patients with advanced heart failure reported reduced activity of the energy dependent uptake-1 activity. ${ }^{17}$

Furthermore, reduced HED retention may indicate loss of neuronal tissue which has become irreversibly damaged by mechanisms of the underlying disease process. Visualisation of neuronal tissue with immunohistochemical methods is difficult and has to be done in experimental animal models, postmortem, or with explanted hearts. To determine whether the scintigraphic findings observed in this study are caused by irreversible loss or potentially reversible functional disturbances of sympathetic neurons, longitudinal studies in patients receiving treatment with a known effect on autonomic activation are necessary.

Earlier studies in dilated cardiomyopathy patients detected a reduction in time domain measures of heart rate variability as a consequence of severity of heart failure, but not, however, an increase of low frequency components of spectral power, which is considered to be a marker of sympathetic modulation. ${ }^{35}$ In this study no correlation could be detected between the PET findings and the calculated time domain parameters of heart rate variability. One possible explanation is the relatively low number of patients with preserved sinus rhythm as a precondition for this calculation. On the other hand, heart rate variability changes and raised plasma noradrenaline concentrations as markers of systemic autonomic stimulation may be independent of cardiac neuronal disturbances. In accordance, Rundqvist and colleagues found that abnormalities of cardiac sympathetic function occur at an earlier stage of heart failure than systemic sympathetic activation. ${ }^{36}$
CLINICAL IMPLICATIONS: MARKER FOR SEVERITY OF HEART FAILURE

In patients suffering from dilated cardiomyopathy, sympathoadrenergic imaging using I-123 MIBG has been evaluated as a diagnostic tool to assess severity of heart failure. I-123 MIBG cardiac retention correlates with severity of heart failure in several studies. Ejection fraction and exercise capacity as well as left ventricular tissue noradrenaline content were related to I-123 MIBG retention..$^{33}{ }^{37-39}$ However, differentiation between various aetiologies of heart failure was not possible with this method. ${ }^{40}$

Application of PET technology, with its superior spatial resolution and the possibility of quantitative evaluation, represents advantages concerning quantification of global and regional changes. Our study is the first to use PET and HED as sympathetic tracer in this patient population. The correlation between the extent of abnormalities of sympathoadrenergic imaging and markers for the severity of heart failure, derived from NYHA functional class and resting ejection fractions, was weak although significant.

SIGNIFICANCE OF REGIONAL SYMPATHETIC DENERVATION FOR PROGNOSIS

The significance of I-123 MIBG cardiac imaging as an independent prognostic marker was demonstrated by Merlet and colleagues ${ }^{41}$ in 90 patients with cardiomyopathy of different origin. I-123 MIBG cardiac retention was a more potent predictor of survival than radionuclide left ventricular ejection fraction, $x$ ray cardiothoracic ratio, and echographic end diastolic diameter. However, interpretation of these findings should be done carefully, as the study population was small and consisted predominantly of patients with advanced heart failure caused by dilated cardiomyopathy. No information was provided on whether MIBG imaging was more useful in predicting sudden cardiac death or progressive heart failure, the main factors influencing prognosis of patients with ischaemic and congestive heart failure. ${ }^{42}$

There may be several explanations for the prognostic significance of autonomic nervous system involvement in cardiac failure. Raised plasma noradrenaline concentrations are related to the severity of left ventricular dysfunction, ${ }^{43-45}$ and indicate the degree of activation of compensatory mechanisms necessary to maintain cardiac output. On the other hand, myocardial failure is associated with functional and structural changes of cardiac autonomous innervation. The presence of regional myocardial sympathetic denervation after myocardial infarction was related to the development of arrhythmia. ${ }^{46}{ }^{47}$ Furthermore, in patients with ventricular tachycardias in the absence of coronary heart disease, regional cardiac sympathetic denervation could be demonstrated using MIBG and SPECT. ${ }^{48}$ Inhomogeneity of electrophysiological properties and an oversensitivity to circulating catecholamines, which might be caused by an upregulation of $\beta$ receptors in the denervated regions, were discussed as possible mecha- 
nisms for the development of arrhythmia. Increased circulating noradrenaline concentrations may act as a trigger for arrhythmogenesis in this setting, ${ }^{49}$ and may cause further deterioration of cardiac function. ${ }^{50}$ This explanation is supported by the finding that $\beta$ blocker treatment reduces the incidence of arrhythmia after myocardial infarction.

\section{STUDY LIMITATIONS}

As mentioned above, this study was not designed to address the prognostic implications of sympathoadrenergic dysfunction in dilated cardiomyopathy. The number of patients was not sufficient, and the observation time too short to gain information on this matter.

Another unsolved problem is the mechanism of neuronal injury in dilated cardiomyopathy. Differentiation between uptake and storage abnormalities is not possible using HED as tracer of cardiac sympathetic innervation. The development of new tracers with different behaviour concerning uptake, storage, and metabolism may provide better tools to answer this question.

\section{Conclusions}

In this study, using PET in combination with HED in patients with dilated cardiomyopathy, global reduction as well as regional abnormalities of cardiac sympathetic tracer uptake, with lowest values in the inferoapical segments, were demonstrated. The degree of abnormality was positively correlated to severity of heart failure. The pathogenetic mechanisms as well as the prognostic significance of these findings is still uncertain. Thus, in patients with dilated cardiomyopathy, sympathoadrenergic imaging with HED should be evaluated as a tool for selecting high risk patients for pump failure or sudden cardiac death.

The excellent technical assistance of C Kruschke, G Schüpferling, E Selecka-Zalkoudek, S Unterweger, and C Kolligs is gratefully acknowledged. The support of the radiochemists and cyclotron staff at the Technische Universität München is greatly appreciated. Part of this study was supported by grant ROIHI 47543 from the National Heart, Lung, and Blood Institute, National Institutes of Health.

1 Francis GS, Goldsmith SR, Levine TB, et al. The neurohumoral axis in congestive heart failure. Ann Intern Med 1984;101:370-7.

2 Leimbach WN, Wallin GB, Victor RG, et al. Direct evidence from intraneural recordings for increased central sympathetic outflow in patients with heart failure. Circulation 1986;73:913-19.

3 Francis GS, Rector TS, Cohn JN. Sequential neurohumoral measurements in patients with congestive heart failure. $A m$ Heasurements in patients

4 Swedberg K, Eneroth P, Kjekshus J, et al. Hormones regulating cardiovascular function in patients with severe congestive heart failure and their relation to mortality. Circulation 1990;82:1730-6.

5 Francis GS, Benedict C, Johnstone DE, et al. Comparison of neuroendocrine activation in patients with left ventricular dysfunction with and without congestive heart failure: substudy of the studies of left ventricular dysfunction (SOLVD). Circulation 1990;82:1724-9.

6 Hasking GJ, Esler MD, Jennings GL, et al. Norepinephrine spillover to plasma in patients with congestive heart failure: evidence of increased overall and cardiorenal sympathetic nervous activity. Circulation 1986;73:615-21.

7 Fowler MB, Laser JA, Hopkins GL, et al. Assessement of the $\beta$-adrenergic receptor pathway in the intact failing human heart: progressive receptor down-regulation and subsensitivity to agonist response. Circulation 1986;74:1290-302.

8 Bristow MR, Ginsburg R, Umans V, et al. $\beta 1$-and

$\beta 2$-adrenergic receptor subpopulations in nonfailing and $\beta 2$-adrenergic receptor subpopulations in nonfailing and
failing human ventricular myocardium: coupling of both receptor subtypes to muscle contraction and selective $\beta 1$-receptor down-regulation in heart failure. Circ Res 1986;59:297-309.

9 Brodde OE. $\beta 1$ - and $\beta 2$-adrenoceptors in the human heart property, function, and alterations in chronic heart failure. Pharmacol Rev 1991;43:203-42.

10 Bristow MR, Hershberger RE, Port JD, et al. Betaadrenergic pathways in nonfailing and failing human ventricular myocardium. Circulation 1990;82:12-25.

$11 \mathrm{Bohm} \mathrm{M}$, Gierschik P, Jakobs KH. Increase of Gi $\alpha$ in human hearts with dilated but not ischemic cardiomyopathy. Circulation 1990;82:1249.

12 Bristow MR, Feldman AM. Changes in the receptor-G protein-adenyl cyclase system in heart failure from various types of heart muscle disease. Basic Res Cardiol 1992;1:1535.

13 Rose CP, Burgess JH, Cousineau D. Reduced aortocoronary sinus extraction of norepinephrine in patients with left ventricular failure secondary to long-standing pressure or volume overload Circulation 1983;68:241-4.

14 Meredith IT, Eisenhofer G, Lambert GW, et al. Cardiac sympathetic nervous activity in congestive heart failure: evidence for increased neuronal norepinephrine release and preserved neuronal uptake. Circulation 1993;88:13645.

15 Port JD, Gilbert EM, Larrabee P, et al. Neurotransmitter depletion compromises the ability of indirect-acting amines to provide inotropic support in the failing human heart. Circulation 1990;81:929-38.

16 Rose CP, Burgess JH, Cousineau D. Tracer norepinephrine kinetics in coronary circulation of patients with heart failure secondary to chronic pressure and volume overload. f Clin Invest 1985;76:1740-7.

17 Bohm M, La Rosée K, Schwinger RHG, et al. Evidence for reduction of norepinephrine uptake sites in the failing reduction of norepinephrine uptake sites in the

18 Schwaiger M, Kalff V, Rosenspire $\mathrm{K}$, et al. Noninvasive evaluation of sympathetic nervous system in human heart by positron emission tomography. Circulation 1990;82: 457-64

19 Schwaiger M, Hutchins GD, Kalff V, et al. Evidence for regional catecholamine uptake and storage sites in the transplanted human heart by positron emission tomography. $\mathcal{F}$ Clin Invest 1991;87:1681-90.

20 Allman KC, Stevens MJ, Wieland DM, et al. Noninvasive assessment of cardiac diabetic neuropathy by carbon-11 hydroxyephedrine and positron emission tomography. $f$ Am Coll Cardiol 1993;22:1425-32.

21 Schwaiger M, Guibourg H, Rosenspire K, et al. Effect of regional myocardial ischemia on sympathetic nervous system as assessed by fluorine-18-metaraminol. 7 Nucl Med 1990;31:1352-7.

22 Schömig A, Dart AM, Dietz R, et al. Release of endogenous catecholamines in the ischemic myocardium of the rat. Par A: locally mediated release. Circ Res 1984;55:689-701.

23 Schömig A, Fischer S, Kurz T, et al. Nonexocytotic release of endogenous noradrenaline in the ischemic and anoxic rat heart: mechanisms and metabolic requirements. Circ Res 1987;60:194-205.

24 Task Force of the European Society of Cardiology and the North American Society of Pacing and Electrophysiology. Heart rate variability: standards of measurement, physiological interpretation, and clinical use. Circulation 1996; 93:1043-65

25 Rosenspire KC, Haka MS, Van DM, et al. Synthesis and preliminary evaluation of carbon-11-metahydroxyephedrine: a false transmitter agent for heart neuronal imaging. F Nucl Med 1990;31:1328-34.

26 Melon P, Schwaiger M. Imaging of metabolism and Melon $P$, Schwaiger $M$. Imaging of metabolism and autonomic innervation of the heart by positr
tomography. Eur $\mathcal{F}$ Nucl Med 1992;19:453-64.

27 Allman KC, Wieland DM, Muzik O, et al. Carbon-11 hydroxyephedrine with positron emission tomography for serial assessment of cardiac adrenergic neuronal function after acute myocardial infarction in humans. $7 \mathrm{Am}$ Coll Cardiol 1993;22:368-75.

28 Calkins H, Allman K, Bolling S, et al. Correlation between scintigraphic evidence of regional sympathetic neuronal dysfunction and ventricular refractoriness in the human heart. Circulation 1993;88:172-9.

29 Melon PG, Nguyen N, DeGrado TR, et al. Imaging of cardiac neuronal function after cocaine exposure using carbon-11 hydroxyephedrine and positron emission tomography. I Am Coll Cardiol 1994;23:1693-9.

30 Wolpers H, Nguyen N, Rosenpire K, et al. C-11 hydroxyephedrine as a marker for neuronal catecholamine retention in reperfused canine myocardium. Coron Artery retention in reperf

31 Rabinovitch MA, Rose CP, Rouleau JL, et al. Metaiodobenzylguanidine [131I] scintigraphy detects impaired myocardial sympathetic neuronal transport function of canine mechanical-overload heart failure. Circ Res 1987;61:797804 .

32 Schofer J, Spielmann R, Schuchert A, et al. Iodine-131meta-iodobenzylguanidine scintigraphy: a noninvasive method to demonstrate myocardial adrenergic nervous system disintegrity in patients with idiopathic dilated cardiomyopathy. $\mathcal{F}$ Am Coll Cardiol 1988;12:1252-8.

33 Glowniak JV, Turner FE, Gray LL, et al. Iodine-123 metaiodobenzylguanidine imaging of the heart in idiopathic congestive cardiomyopathy and cardiac transplants. $₹$ Nucl Med 1989;30:1182-91

34 DeGrado TR, Hutchins GD, Toorongian SA, et al. Myocardial kinetics of carbon-11-meta-hydroxyephedrine: reten- 
tion mechanisms and effects of norepinephrine. $\mathcal{F ~ N u c l ~ M e d ~}$ 1993:34:1287-93.

35 Kienzle MG, Ferguson DW, Birkett CL, et al. Clinical, hemodynamic and sympathetic neural correlates of heart rate variability in congestive heart failure. Am $\mathcal{F}$ Cardiol 1992;69:761-7

36 Rundqvist B, Elam M, Bergmann-Sverrisdottir Y, et al. Increased cardiac adrenergic drive procedes generalized sympathetic activation in human heart failure. Circulation 1997;95:169-75.

37 Glowniak JV, Turner FE, Gray LL, et al. Iodine-123 metaiodobenzylguanidine imaging of the heart in idiopathic congestive cardiomyopathy and cardiac transplants. $\mathcal{F} \mathrm{Nucl} \mathrm{Med}$ 1989;30:1182-91.

38 Simmons WW, Freeman MR, Grima EA, et al. Abnormalities of cardiac sympathetic function in pacing-induced dine scintigraphy. Circulation 1994;89:2843-51.

Takatsu $\mathrm{H}$, Uno Y, Fujiwara

left ventricular iodine-125-MIBG accumulation in cardiomyopathic syrian hamsters using the renin-angiotensin system. $₹$ Nucl Med 1995;36:1055-61.

40 Glowniak JV. Cardiac studies with metaiodobenzylguanidine: a critique of methods and interpretation of results. F Nucl Med 1995;36:2133-7.

41 Merlet P, Valette H, Dubois RJ, et al. Prognostic value of cardiac metaiodobenzylguanidine imaging in patients with heart failure. F Nucl Med 1992;33:471-7.

42 Goldman S, Johnson G, Cohn JN, et al. Mechanism of death in heart failure: the vasodilator-heart failure trials. The V-HeFT VA cooperative studies group. Circulation 1993; 87(suppl 4):24-31
43 Levine TB, Francis GS, Goldsmith SR, et al. Activity of the sympathetic nervous system and renin-angiotensin system assessed by plasma hormone levels and their relationship to hemodynamic abnormalities in congestive heart failure. Am $\mathcal{F}$ Cardiol 1982;49:1659-66.

44 Cohn JN, Levine TB, Olivari MT, et al. Plasma norepinephrine as a guide to prognosis in patients with chronic congestive heart failure. N Engl f Med 1984;311:819-23.

45 Francis GS, Cohn JN, Johnson GR, et al, for the V-HeFT VA Cooperative Studies Group. Plasma norepinephrine, plasma renin activity, and congestive heart failure: relations to survival and the effects of therapy in V-HeFT II. Circulation 1993;87(suppl 4):40-8.

46 Zipes DP. Influence of myocardial ischemia and infarction on autonomic innervation of the heart. Circulation 1990;82: 1095-105.

47 Zipes DP. Sympathetic stimulation and arrhythmias. N Engl F Med 1991;325:656-7.

48 Mitrani RD, Klein LS, Miles WM, et al. Regional cardiac sympathetic denervation in patients with ventricular tachycardia in the absence of coronary artery disease. $7 \mathrm{Am}$ Coll Cardiol 1993;22:1344-53.

49 Meredith IT, Broughton A, Jennings GL, et al. Evidence of a selective increase in cardiac sympathetic activity in patients with sustained ventricular arrhythmias. $N$ Engl $7 \mathrm{Med}$ 1991;325:618-24.

50 Mann DL, Kent RL, Parsons B, et al. Adrenergic effects on the biology of the adult mammalian cardiocyte. Circulation 1992;85:790-804.

\section{IMAGES IN CARDIOLOGY}

\section{Right coronary artery to coronary sinus fistula}

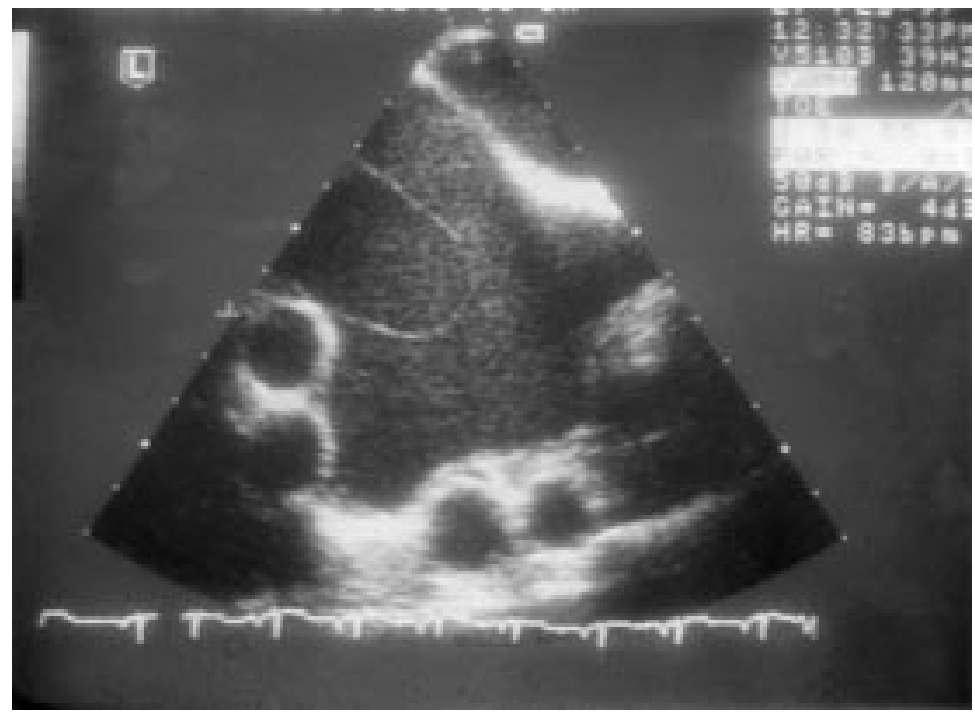

An asymptomatic, 49 year old, white male was noted to have mild cardiomegaly on routine chest radiography. Transthoracic echocardiography revealed a prominent right sinus of Valsalva and an ill defined mass that appeared to be in the right atrium. Transoesophageal echocardiography showed a large vascular structure in the right atrial (RA) wall and an enlarged coronary sinus (CS). Aortography confirmed the presence of a large coronary-cameral fistula. During surgical obliteration it was found to originate at the right coronary artery sinus and empty into the coronary sinus, just proximal to its entry into the right atrium.

C G MISSOURIS

D FLUCK $S$ BRECKER 